\title{
Single-Step Bio-friendly Synthesis of Surface Modifiable, Near-Spherical Gold Nanoparticles for Applications in Biological Detection and Catalysis
}

Vivek D Badwaik ${ }^{\dagger}$, Jane J Bartonojo ${ }^{\dagger}$, Jesse W Evans ${ }^{\dagger}$, Shivendra V Sahi ${ }^{\dagger}$, Chad B Willis ${ }^{\dagger}$, and Rajalingam Dakshinamurthy ${ }^{\dagger} *$

${ }^{\dagger}$ Department of Chemistry, ${ }^{\ddagger}$ Department of Biology, Western Kentucky University, Bowling Green, KY 42101

Phone: 270-745-2136; Fax: 270-745-5361; E-mail: rajalingam.dakshinamurthy@wku.edu

\section{Supporting Information}

This document contains a table S1 showing the ingredients used to prepare the medium for the synthesis of dextrose assisted GNPs. Also TEM images and particle size histogram generated using EDS 2000, version 2.6 software for three different sizes of GNPs are shown in figure S1. Elemental composition of the GNPs were detected by energy dispersive X-ray (EDS) as shown in figure S2a. The interaction between the GNPs and dextrose molecules were analyzed by comparing the FTIR spectrum of free dextrose and the GNPs capped with dextrose as shown in figure $S 2 b$. 
Table S1: Ingredients in the aqueous reaction medium used for the synthesis of GNP's

\begin{tabular}{|l|l|}
\hline \multicolumn{1}{|c|}{ Ingredients } & $100 \mathrm{ml}$ \\
\hline $\mathrm{KH}_{2} \mathrm{PO}_{4}$ & 2.6 grams \\
\hline $\mathrm{K}_{2} \mathrm{HPO}_{4}$ & 2.0 grams \\
\hline $\mathrm{Na}_{2} \mathrm{HPO}_{4}$ & 1.8 grams \\
\hline $\mathrm{K}_{2} \mathrm{SO}_{4}$ & 0.48 grams \\
\hline Dextrose & 0.1 grams \\
\hline $\mathrm{KAuCl}$ & $\begin{array}{l}\text { appropriate } \\
\text { concentrations }\end{array}$ \\
\hline
\end{tabular}




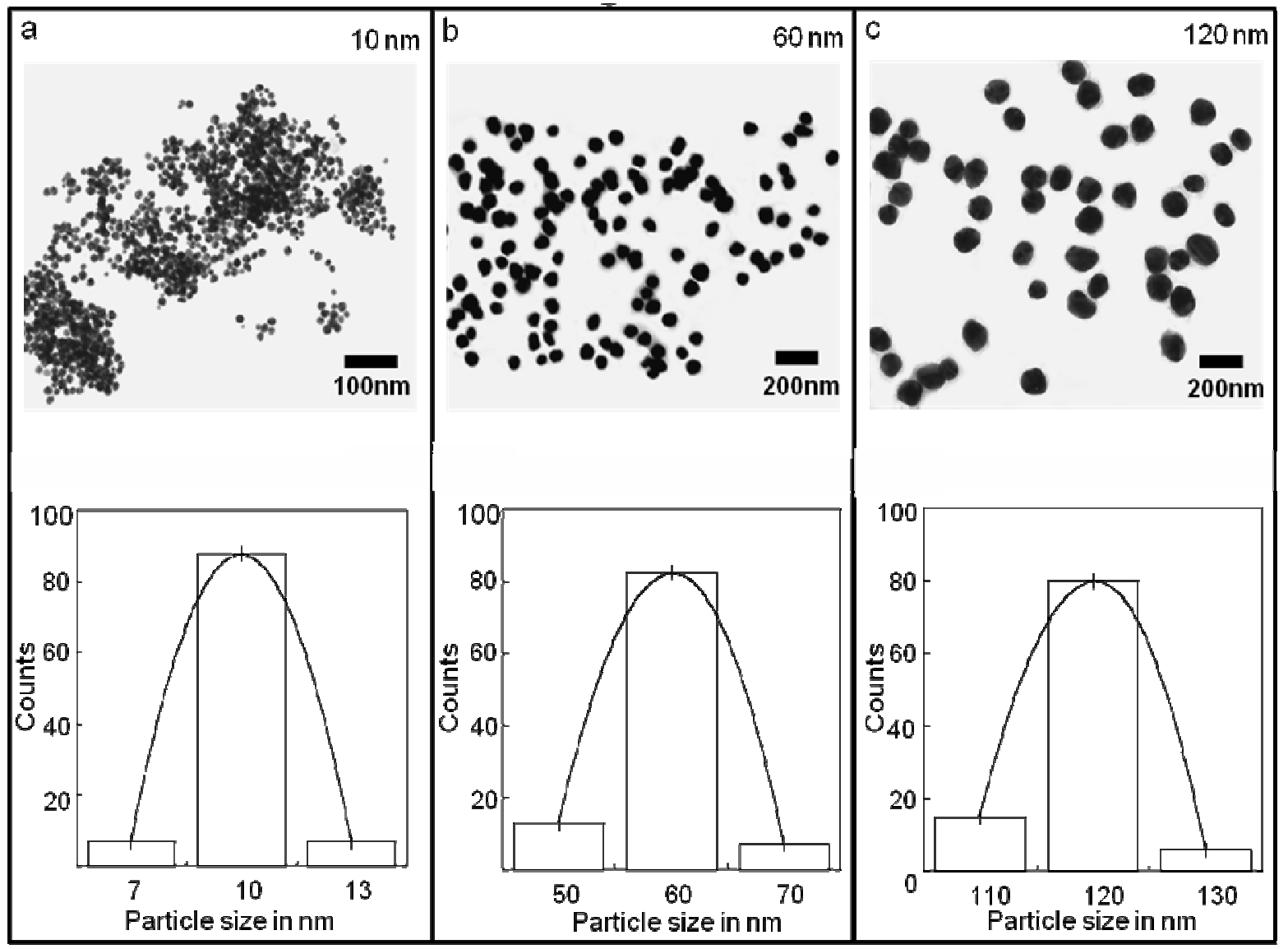

Figure S1. Representative TME images of GNPs of three particles sizes along with the corresponding histogram of the particle size distribution. (a) $10 \mathrm{~nm}$ (scale bar = $100 \mathrm{~nm}$ ), (b) 60 $\mathrm{nm}($ scale bar $=200 \mathrm{~nm}),($ c) $120 \mathrm{~nm}($ scale bar $=200 \mathrm{~nm})$. 


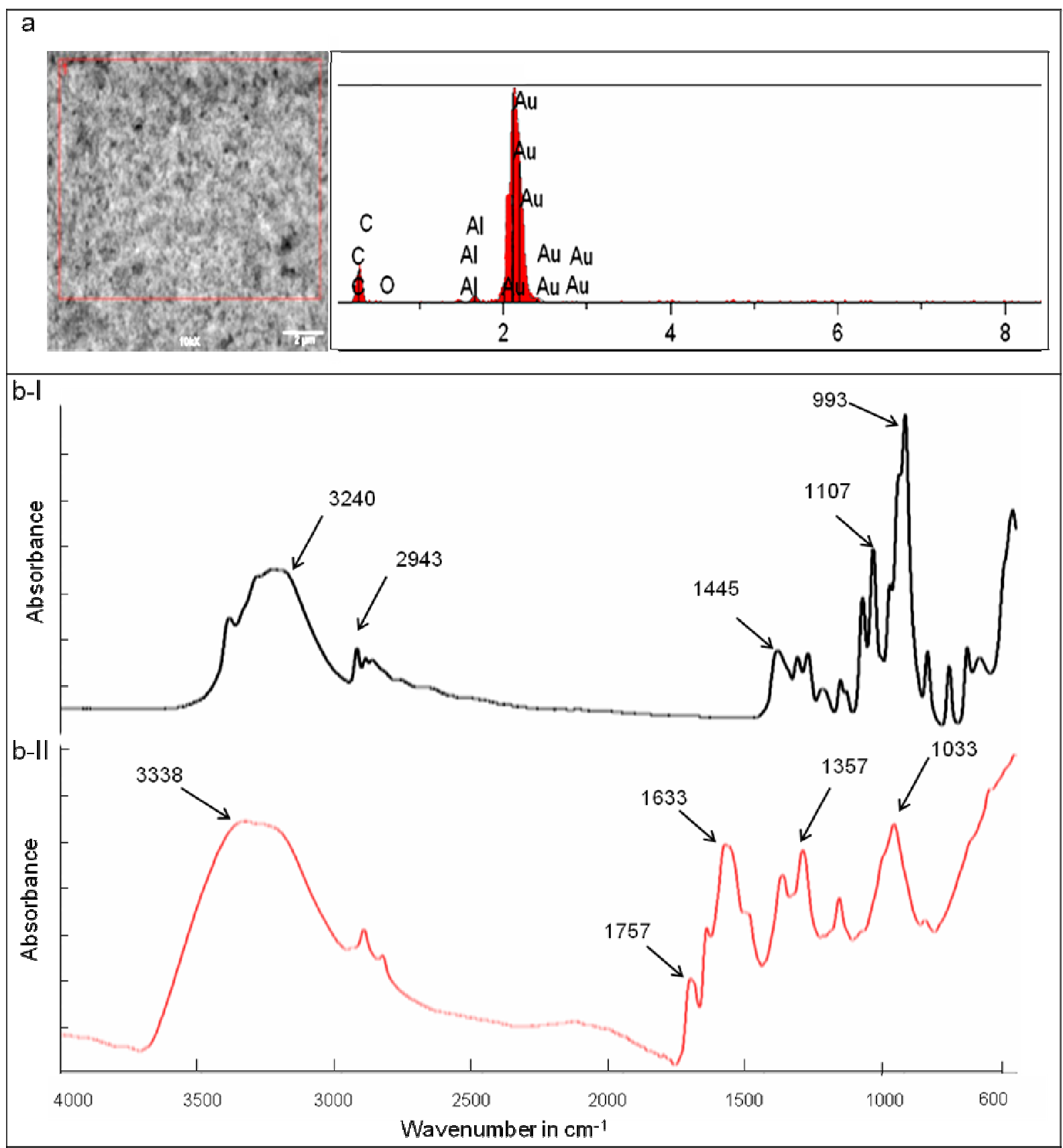

Figure S2. EDS spectrum and FTIR. (a) EDS spectrum of dextrose stabilized GNPs, (b-I) FTIR spectra of neat dextrose, (b-II) FTIR spectra of dextrose stabilized GNPs. 


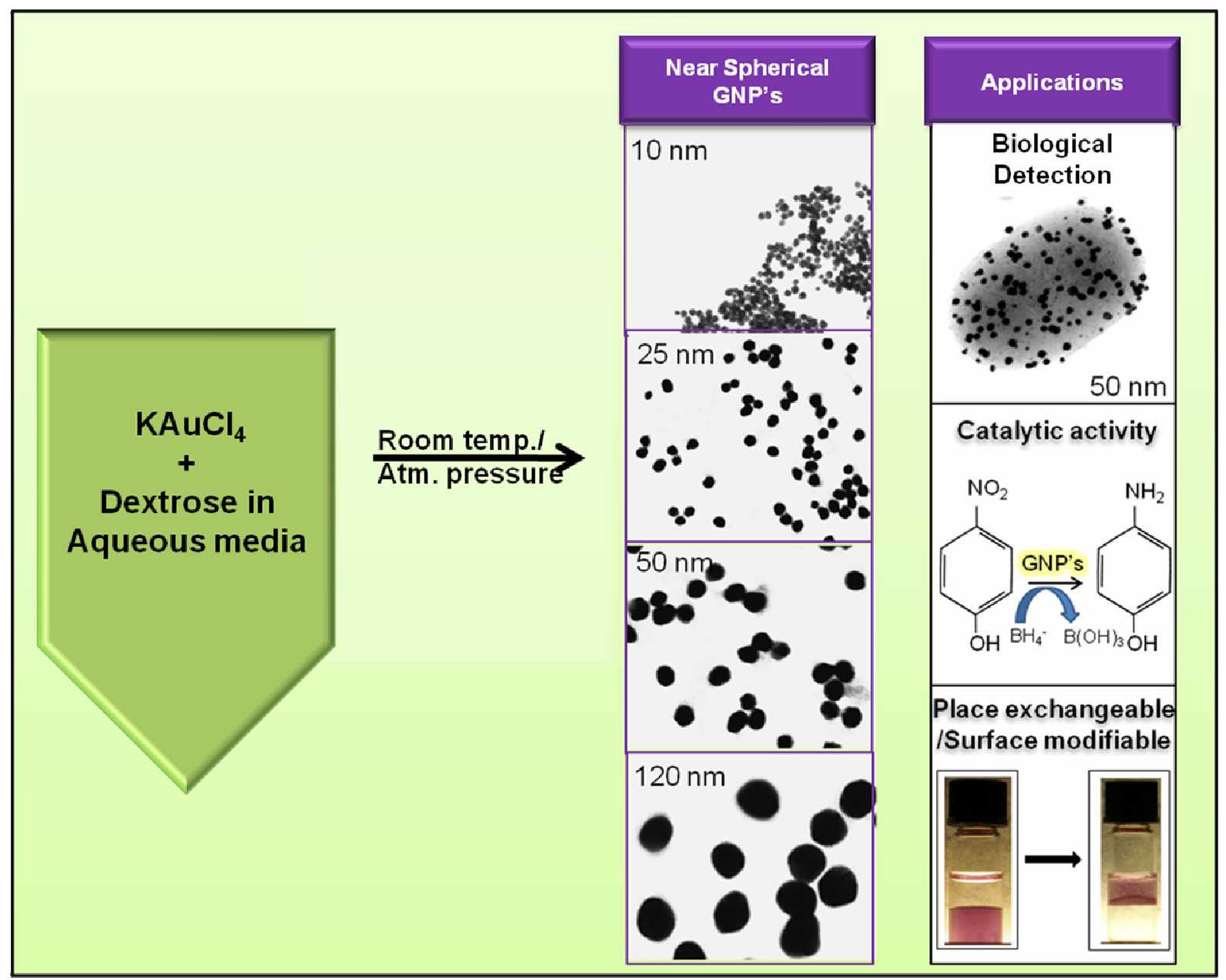

Figure S3. Schematic Representation of the Environment-Friendly Single Step Aqueous Synthesis of GNPs and its Applications. 